\title{
Development and Application of the Single-Spiral Inductive-Capacitive Resonant Circuit Sensor for Wireless, Real-Time Characterization of Moisture in Sand
}

\author{
Andrew J. DeRouin, ${ }^{1}$ Zhanping You, ${ }^{2}$ Morgan Hansen, ${ }^{2}$ \\ Aboelkasim Diab, ${ }^{2}$ and Keat Ghee Ong ${ }^{1}$ \\ ${ }^{1}$ Department of Biomedical Engineering, Michigan Technological University, Houghton, MI 49931, USA \\ ${ }^{2}$ Department of Civil and Environmental Engineering, Michigan Technological University, Houghton, MI 49931, USA
}

Correspondence should be addressed to Keat Ghee Ong; kgong@mtu.edu

Received 12 July 2013; Revised 6 September 2013; Accepted 8 September 2013

Academic Editor: Eugenio Martinelli

Copyright (C) 2013 Andrew J. DeRouin et al. This is an open access article distributed under the Creative Commons Attribution License, which permits unrestricted use, distribution, and reproduction in any medium, provided the original work is properly cited.

A wireless, passive embedded sensor was designed and fabricated for monitoring moisture in sand. The sensor, consisted of an inductive-capacitive (LC) resonant circuit, was made of a printed spiral inductor embedded inside sand. When exposed to an electromagnetic field, the sensor resonated at a specific frequency dependent on the inductance of the inductor and its parasitic capacitance. Since the permittivity of water was much higher than dry sand, moisture in sample increased the parasitic capacitance, thus decreasing the sensor's resonant frequency. Therefore, the internal moisture level of the sample could be easily measured through tracking the resonant frequency using a detection coil. The fabrication process of this sensor is much simpler compared to LC sensors that contain both capacitive and inductive elements, giving it an economical advantage. A study was conducted to investigate the drying rate of sand samples of different grain sizes. The experimental data showed a strong correlation with the actual moisture content in the samples. The described sensor technology can be applied for long term monitoring of localized water content inside soils and sands to understand the environmental health in these media, or monitoring moisture levels within concrete supports and road pavement.

\section{Introduction}

Monitoring of water content in porous mechanical structures is important in ensuring and predicting their mechanical integrity. For example, monitoring water content in asphalt is critical for assessing its structural stability because the bond between asphalt and aggregate particles can be broken or reduced in strength due to the presence of unwanted moisture. Similarly, it is important in concrete mixing to take into consideration the natural moisture of aggregates during the mix design to ensure proper slump and compressive strength of the resulting concrete structure [1].

Several methods currently exist for measuring water content in civil constructs. A common method involves determining the mass of a sample before and after drying. The disadvantages of this method include the need to remove the material (or a sample), the length of the drying process, and the possibility of degrading the sample as a result of heating. Additional complications can arise when moisture-sensitive environments must be breeched to collect the sample for drying. A more sophisticated method for measuring water content consists of two insulated conductor probes. The permittivity of the medium will depend on water content between the probes and can be determined by measuring the capacitance between the probes using radio or microwave energy [2]. This method allows for a direct measurement of the sample without the need of removing it, but its accuracy can depend on the amount of samples available and closed environments must still be breached to take the measurement.

Thermographic measurement techniques have also been employed for measurement of moisture content in soils and 


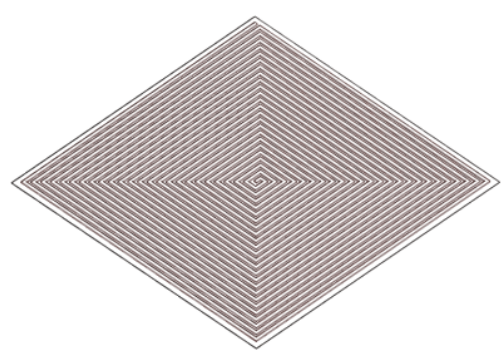

(a)

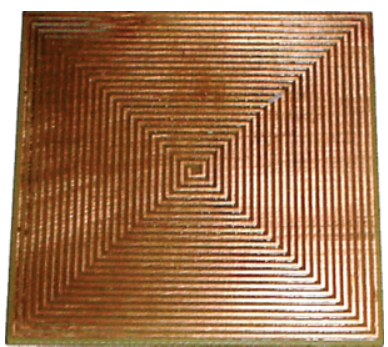

(b)

FIGURE 1: The (a) illustration and (b) photograph of the sensor, which was comprised of a single-layer, spiral inductor circuit printed on a substrate.

building materials $[3,4]$. This technique generally utilizes infrared sensing methods to determine heat transfer rates on a surface, which can then be used to approximate the moisture content of the surface in question. While this approach provides a novel method of moisture measurement, the surface of interest must be changing in temperature. Moreover, the depth that this method can measure into the imaged medium is extremely limited, making it unsuitable for large batch measurements. Furthermore, this detection method is possible only if there is a line-of-sight path from the detector to the sample, rendering it ineffective when the sample is contained or covered by materials that block infrared light.

An embedded moisture monitoring sensor based on a planar, printed spiral inductor was developed. The sensor represented a new design of inductor-capacitor (LC) resonant circuit sensors, which have previously been proposed for moisture monitoring in civil engineering materials [5-10]. The water-sensing component of the sensor is similar to many capacitive humidity sensors [11-13], which are based on changes in the capacitance of a capacitor that in turn shifts the resonant frequency of the LC circuit. As water accumulates on the capacitor's surface, the effective permittivity of the medium at the proximity of the capacitor increases significantly due to the high permittivity of water (about 70-80) compared to air (about 1).

Figure 1 illustrates the new sensor, which consisted of only a square spiral inductor. To prevent excessive water from shorting the inductor, an electrical insulating coating was applied on top of the sensor. Similar to other LC sensors, the new sensor is lightweight, low cost, robust, and portable. This sensor requires neither any direct physical connections between the sensor and the data processing electronics nor the precise alignment between sensor and detector needed in optical telemetry. This makes the sensor very useful in operations such as construction, mining, and agriculture applications where the monitoring sites are difficult to access. The sensor is completely passive, so there are no battery lifetime problems. For applications such as roadway condition monitoring, the LC sensor is affordable due to its low cost compared to other highway monitoring sensors [14-16]. In terms of reliability, this technology can be superior to other passive, wireless sensors based on the radio frequency identification device (RFID) technology [17-19] due to its simple design, which is less prone to failure than the electrical circuit components and ICs found in the RFID sensors. Due to its flexibility and wireless, passive nature, the LC sensor technology can be applied in a wide range of civil infrastructures such as asphalt pavement, concrete bridges, pavement substructures, pile structures, and hydraulic structures.

An LC sensor was developed to monitor water content in sand materials. The sensors were embedded in the sand samples so the internal water content of the samples could be remotely measured with a detection coil by tracking the changes of the sensor's resonant frequency [20]. Although the operating mechanism of the new sensor is similar as the previously reported LC sensors [20], the new design only requires a single layer of spiral inductor instead of multiple layers in the old design for completing the inductorcapacitor circuit. In the new design, the parasitic capacitance of the spiral inductor acts as the sensing element. As a result, its fabrication process is simplified resulting in a lower fabrication cost and ultimately a lower sensor unit cost. Because many applications of this sensor technology would require wide area monitoring, which utilizes a large number of sensors, the new sensor can present a significant cost saving.

\section{Experiments}

2.1. Sensor Description. The sensor was embedded inside the test sample to monitor water content in situ. The sensor's resonant frequency was wirelessly interrogated by measuring the impedance of a circular detection coil placed at a short distance from the sensor. Prior to measuring the sensor response, the initial impedance is measured with no sensor present, and is then subtracted from all future measurements. Figure 2 shows the typical background-subtracted sensor's impedance, where the resonant frequency $f_{0}$ is defined as the frequency at the maximum of the real part of the impedance (resistance). The resonant frequency of the sensor is related to the inductance $L$ and parasitic capacitance $C$ of the spiral inductor as given [12]

$$
f_{0}=\frac{1}{2 \pi \sqrt{L C}} .
$$

The parasitic capacitance of the inductor is dependent on its dimension and the permittivity of the medium in 


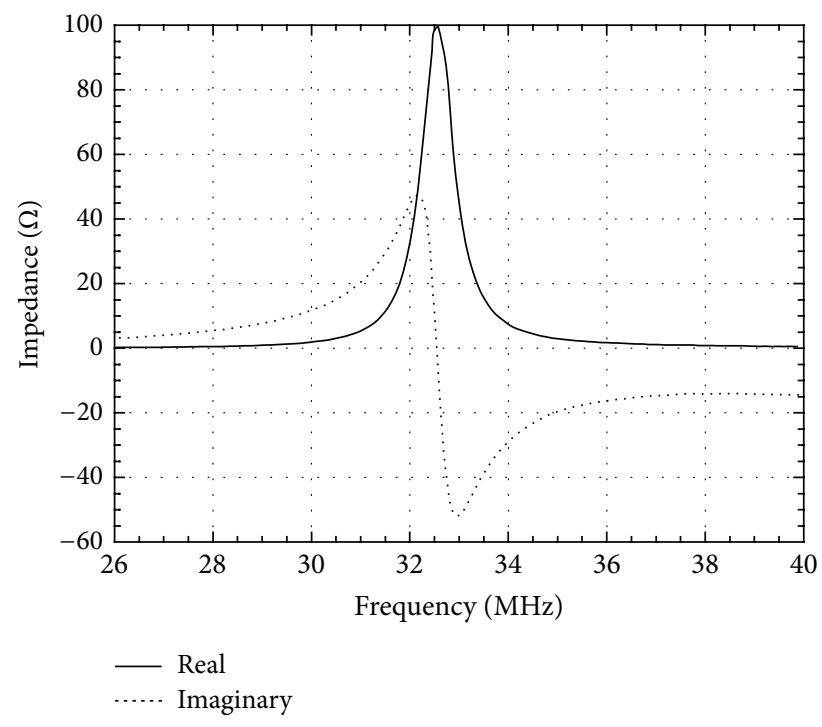

FIgURE 2: The real and imaginary portions of the impedance spectrum of the sensor after the background subtraction. The resonant frequency $f_{0}$ is defined as the maximal of the real impedance.

the proximity of the sensor. The relative permittivity of water, about 70-80, is much higher than that of most civil construction materials, which are generally less than 5 [21]. As a result, the presence of water near the sensor significantly increases the capacitance of the capacitor, leading to a reduction in the sensor's resonant frequency according to (1). Since the inductance is constant throughout the experiment, (1) indicates that the resonant frequency of the sensor is purely dependent on the capacitance, so it is used to track permittivity variations.

2.1.1. Sensor Fabrication. The sensor was made of a singlesided FR-4 fiberglass printed circuit board (PCB). The outer diameter of the square, twenty-four turn spiral inductor was $50 \mathrm{~mm}$. The width of each trace was identical to the spacing between two adjacent electrodes, which was $0.5 \mathrm{~mm}$. The sensor was milled using a CNC milling machine. A layer of $700 \mu \mathrm{m}$ thick enamel (Rustoleum clear enamel) was coated on the sensor to prevent excessive water from shorting the circuit. The resonance of the as-fabricated sensor is plotted in Figure 2, where the resonant frequency is determined as the frequency that corresponds to the peak of the real impedance. It should be noted that this sensor design is not symmetric, and as a result, the effective permittivity will be a weighted average of the fiberglass material and that of the media surrounding the sensor. Thus, the sensitivity to moisture changes will be greater on one side than the other. This can be compensated by improved sensor designs consisting of a thinner backing material or by using a single piece of rigid conductive material. This attribute can also be utilized to control the sensitivity and range of moisture the sensor can measure by applying coatings of various thicknesses over the conductive traces and thereby averaging down the contribution of water to the overall permittivity near the sensor.
TABLE 1: The sand particle size for each sand sample.

\begin{tabular}{lcc}
\hline Sand Sample & $\begin{array}{c}\text { Particle size }(\mathrm{mm}) \\
\text { distribution }\end{array}$ & $\begin{array}{c}\text { Particle size }(\mathrm{mm}) \text { in } \\
\text { record }\end{array}$ \\
\hline Sample A & $0.15-0.3$ & 0.3 \\
Sample B & $0.3-0.6$ & 0.6 \\
Sample C & $0.6-1.18$ & 1.18 \\
\hline
\end{tabular}

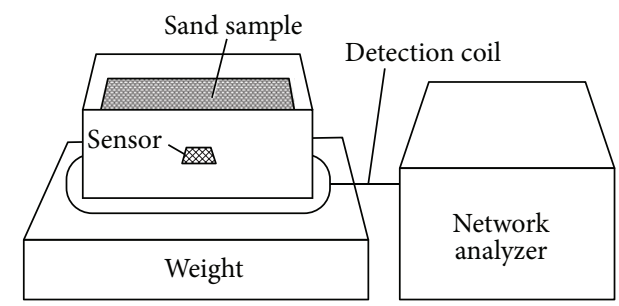

Figure 3: The experimental setup. The test sample was placed inside a plastic container on top of the detection coil. The electrical impedance of the detection coil was measured with an impedance analyzer.

2.2. Experimental Setup. An experiment was conducted to monitor the water content in sand samples. The natural sand samples (mainly silicon dioxide) were obtained from a pavement construction project site in Michigan. The sand samples were dried and then sieved to various sizes with standard sieve shakers. For this study, three samples were used and each sample represented one range of particle sizes, that is, Sample A, Sample B, and Sample C, each containing particle sizes ranging from 0.15 to $0.3 \mathrm{~mm}, 0.3$ to $0.6 \mathrm{~mm}$, and 0.6 to $1.18 \mathrm{~mm}$, respectively, were used in the experiment as seen in Table 1 . After adding water, the dampened sand was weighed to determine the total mass of water and sand. The weight of dry sand was determined by measuring the sample after drying and before adding water. As shown in Figure 3, a plastic container with a crosssectional area of $10 \mathrm{~cm} \times 10 \mathrm{~cm}$ was then filled with the sand up to $1 \mathrm{~cm}$. The sensor was placed at the center of the sample with conductive traces facing upward, and additional sand was added until the total height of the sand was $2 \mathrm{~cm}$. The detection coil was adhered to the bottom of the plastic container and remained secured through all experiments. Additionally, the mass of the sample was tracked throughout the experiment to compare to the sensor readings. An Agilent Spectrum/Network Analyzer 4396B was used to measure the sensor impedance at source power of $1 \mathrm{~mW}$. The maximum measurement error for all experiments was determined as $300 \mathrm{kHz}$. The error was determined by measuring the fluctuation off the resonant frequency while the sensor was stabilized in dry air. A customized computer program, developed in Microsoft Visual Basic, was used to control the operation of the impedance/spectrum analyzer through the General Purpose Interface Bus. Prior to each experiment, a background subtraction routine was performed on the impedance of the coil in the absence of the sensor. The mass of the sample was recorded with a digital scale over time under a fairly constant temperature and relative humidity 


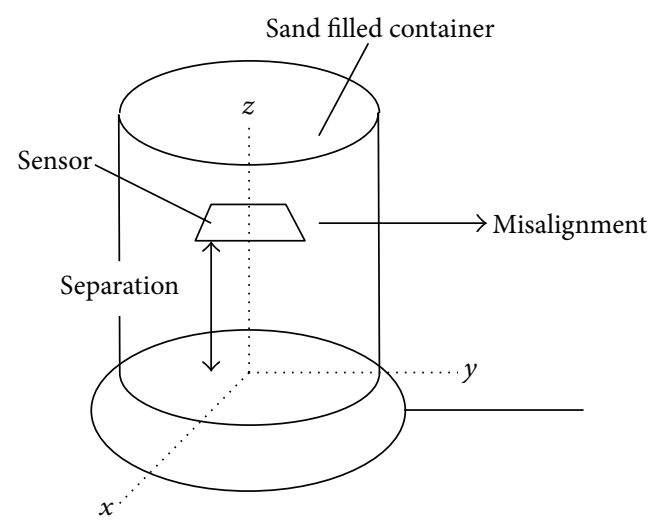

FIGURE 4: Experimental setup for evaluating the signal reduction due to separation and alignment between the sensor and coil. Separation distance was defined as the distance between the sensor and coil when both were on the same axis ( $z$ axis). The misalignment was defined as the movement of the sensor away from the $z$ axis along the $y$ axis.

of $20.0-22.2^{\circ} \mathrm{C}$, and between 45 and $55 \%$ relative humidity, respectively. The ventilation of the room was $0.6 \mathrm{~m}^{3} / \mathrm{s}$.

To determine the potential detection range of the sensor, the signal strength of the sensor was measured when its location with respect to the coil changed. The setup used for this experiment was illustrated in Figure 4, where the sensor was embedded in the middle of a cylinder container filled with sand. The detection coil was placed at the bottom of the container. Both dry and wet sand samples with $0.6 \mathrm{~mm}$ grain size were used to simulate the monitoring condition for dry and wet samples. The separation distance was increased by adding sand to the desired distance, placing the sensor on the sand and then adding $2 \mathrm{~cm}$ of sand on top. To determine the allowable misalignment between the sensor and the coil, the sensor was embedded at the desired separation distance, and the whole container was moved from the center alignment on the surface of the coil as illustrated in Figure 4 (perfect alignment was defined when the center of the sensor and the center of the coil were on the $z$ axis). For all experiments, a single-loop, $10.5 \mathrm{~cm}$ diameter coil was used to interrogate the sensor. Because the detection range depends on the coupling of the sensor to the detection coil, this range will vary depending on both sensor and coil dimensions.

\section{Results and Discussion}

3.1. Monitoring of Water Content in Sand Samples. Using the experimental setup shown in Figure 4, the change in the sensor's resonant frequency was measured as a function of time. Three samples with particle size A started with an average water mass of $37.0 \mathrm{~g}$ and an average total mass of $350.7 \mathrm{~g}$. The dampened sand was left to dry for at least 70 hours while the analyzer measured the change in resonant frequency. The same procedure was used for Sample B and Sample C. Table 2 lists the average initial mass and water mass for all samples.

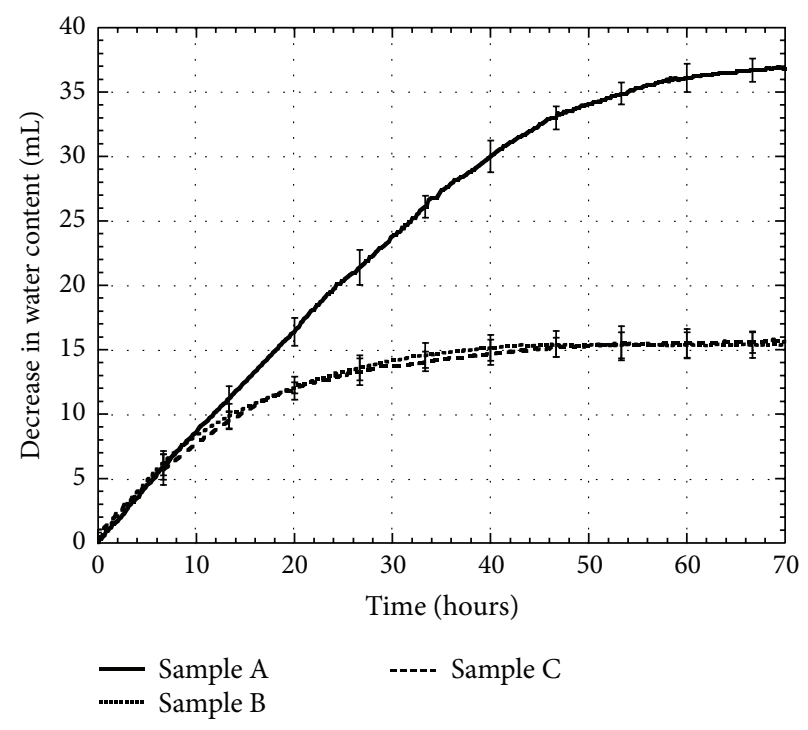

FIGURE 5: The decrease in water content in sand samples during drying of with the size of the sand ranging from 0.15 to $2.36 \mathrm{~mm}$ standard deviations of the curves are shown as error bars in the plot.

Figure 5 plots the moisture content in sand samples obtained from the change in mass of the samples over time. Sample A, with average particle size of $0.3 \mathrm{~mm}$, soaked up the highest amount of water correlating to about $11.8 \%$ of the mass of sand, which was more than twice that of Sample B and Sample C. As a result, water evaporation rate for Sample A was much lower than that for Sample B and Sample C. The characteristic curves depicted in the figure correlate to the expected behavior of sand drying under convectional drying conditions as given in [22]. Ideally, the first phase of drying includes a linear relationship corresponding to surface evaporation followed by an exponentially decaying region related to capillary action in the sand.

Figure 6 illustrates the typical change in the sensor's resonant frequency over time. The resonant frequency of the sensor increased over time, indicating the drying of the sample. The curves showed a steady increase as the water was evaporating until reaching equilibrium when the sand samples were dry. This is expected because the presence of moisture would increase the overall permittivity of the sand. Since resonant frequency was inversely proportional to the permittivity, it increased as water started to evaporate. It is evident that the grain size of the sand samples affects the sensor response. Similar to the water evaporation curves in Figure 5, it took more than 60 hours for the sensor's resonant frequency curve to saturate for Sample A, as compared to the curves for Sample B and Sample C which took about 30 hours to saturate.

Figure 7 shows a linear correlation between the changes in water and the resonant frequency response. As shown, the resonant frequency of the sensor decreased with increasing water content. The response of the sensor was very similar in all sand samples.

Experiments were performed to investigate the effect of the position and distance of the coil with respect to the sensor. 
TABLE 2: The average initial mass and water absorbed for each sand sample. Numbers in parenthesis are standard deviations of measurements.

\begin{tabular}{lccc}
\hline Sand sample & Water mass $(\mathrm{g})$ & Total mass $(\mathrm{g})$ & Water-to-sand ratio \\
\hline Sample A & $37.0(1.5)$ & $350.7(11.6)$ & $11.8 \%$ \\
Sample B & $15.4(1.1)$ & $336.1(9.0)$ & $4.8 \%$ \\
Sample C & $15.8(0.8)$ & $392.1(8.4)$ & $4.2 \%$ \\
\hline
\end{tabular}

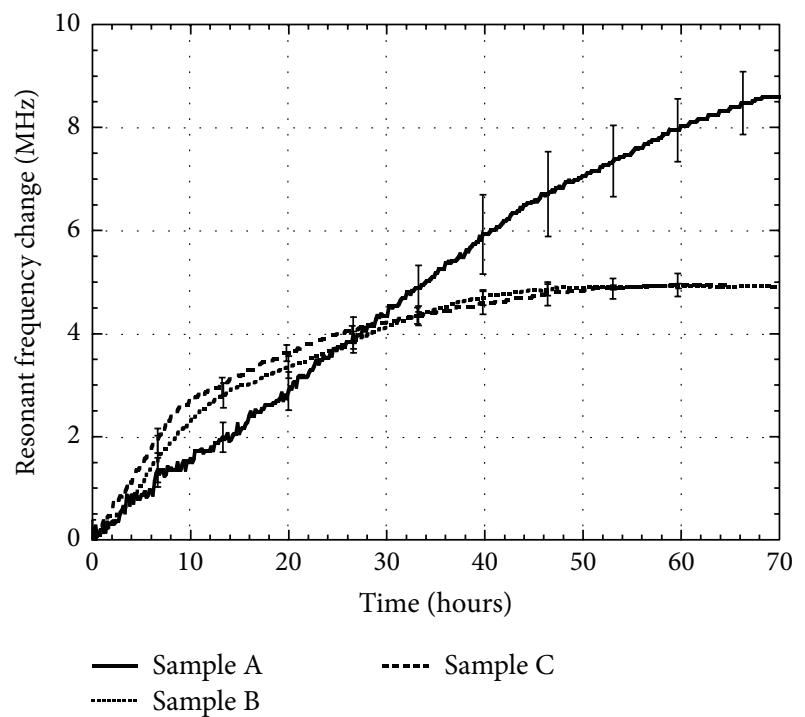

FIGURE 6: A typical sensor's resonant frequency response with respect to time during the drying of the sand sample with the size of the sand ranging from 0.15 to $0.3 \mathrm{~mm}$. Standard deviations of the curves are shown as error bars in the plot.

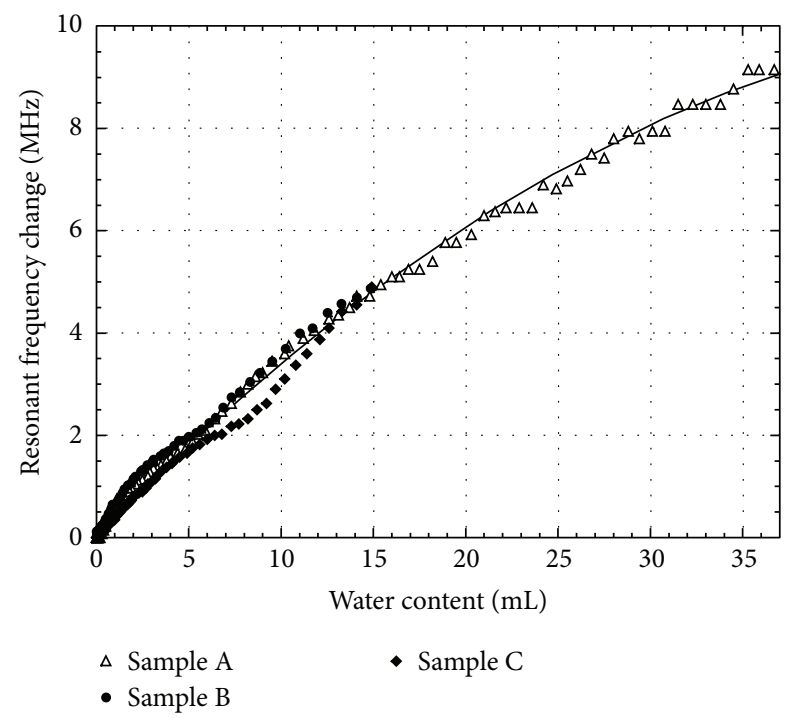

Figure 7: The change in the sensor's resonant frequency as a function of decreasing water content in sand samples. Note that the $x$ axis of the plot is the water content in sand ( 0 means no water in sand), as opposed to the $y$ axis of Figure 5, which is the decrease in water content from the saturation level ( 0 means maximum water in sand).

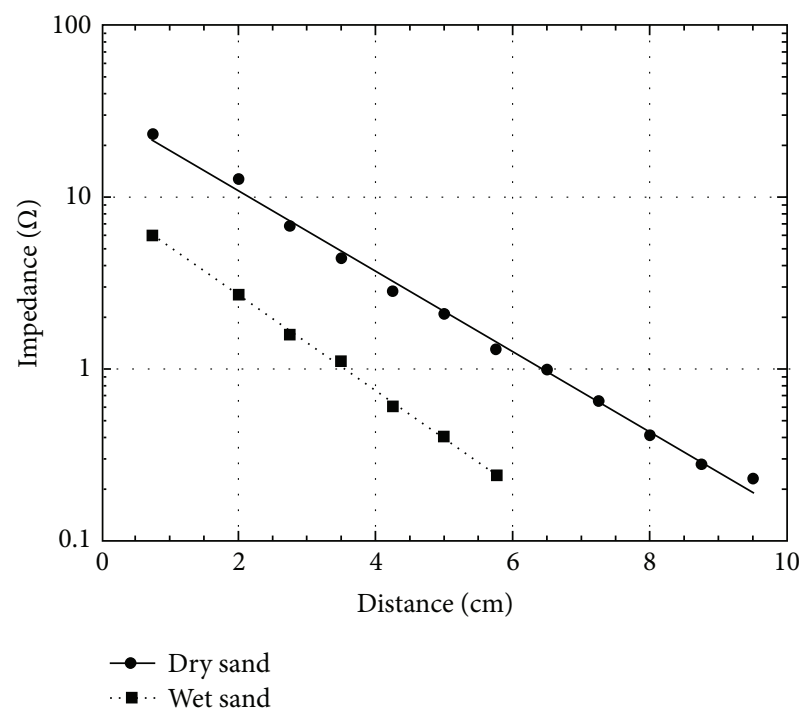

FIgURE 8: Real impedance of the sensor when the separation distance between the sensor and coil varied.

While results indicated no significant change in resonant frequency (less than $100 \mathrm{kHz}$ ), the signal amplitude of the sensor decreased as the separation distance between the sensor and detection coil increased, or the alignment between them changed. As a result, to determine the maximum distance where the sensor can be accurately monitored, an experiment was conducted to measure the variations of the sensor's signal strength due to the increasing separation distance and misalignment from the coil.

As shown in Figure 8, when the sensor and coil were aligned (the center of the sensor and the center of the coil are along the same axis), the maximum separation distance between the sensor and the coil was $9.5 \mathrm{~cm}$ to maintain a signal-to-noise ratio of at least 2 in dry sand (signal-tonoise was determined as the maximum signal amplitude of the sensor over the maximum noise value). The maximum separation distance of the sensor was reduced to $6 \mathrm{~cm}$ when monitoring wet sand due to attenuation of electromagnetic energy in the moisture of wet sand. The sensor can also function with some misalignment from the coil. As shown in Figure 9(a), the sensor signal decreased when its alignment decreased, and the sensor signal decreased more rapidly with increasing separation distance. As depicted in Figure 10, the sensor could maintain a signal-to-noise ratio of 2 with misalignment of up to about $6 \mathrm{~cm}$ when the separation 


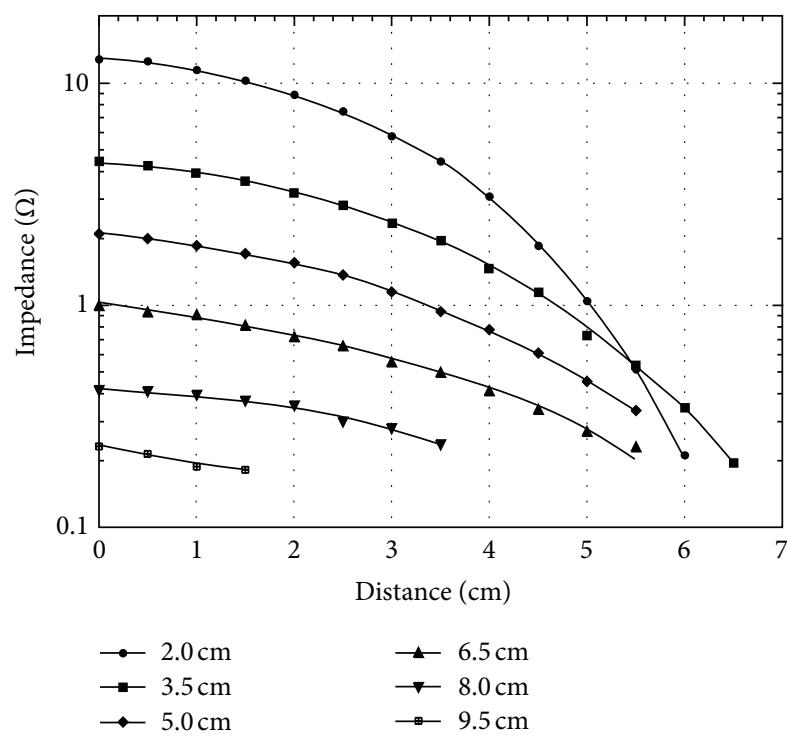

(a)

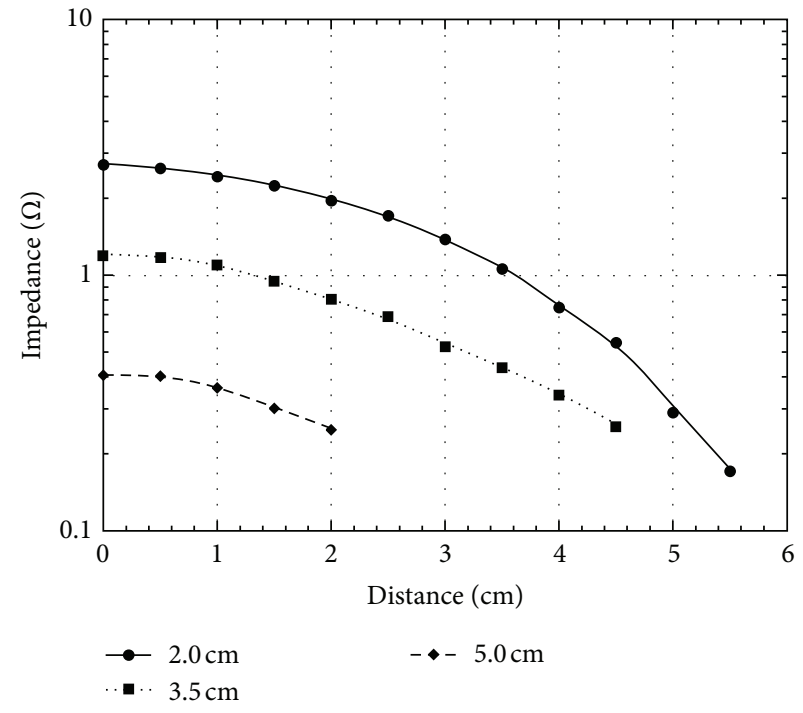

(b)

FIGURE 9: The variation of the sensor's real impedance as a function of misalignment between the sensor and coil when the sensor was in (a) dry sand and (b) wet sand.

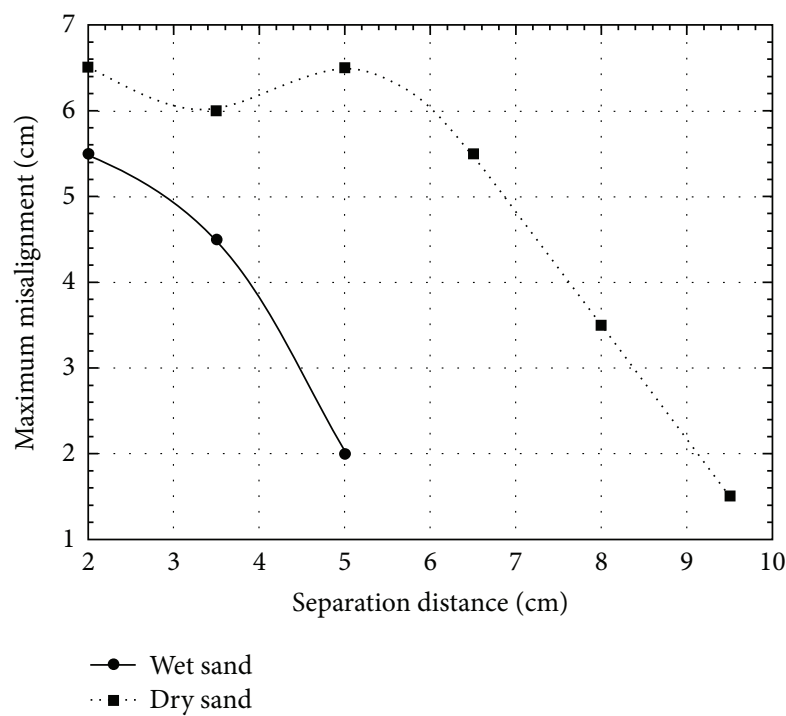

Figure 10: The maximum allowable misalignment between the sensor and coil at different separation distances.

was less than $5 \mathrm{~cm}$, but the misalignment caused a more rapid decrease when the separation was larger. Similarly, the tolerance for misalignment decreased when the sensor was in wet sand (see Figures 9(b) and 10). The detection range can be further improved by increasing the sensor size and increasing the power through the detection coil. In practical applications, the detector location can also be varied until a position is reached having the maximum amplitude.

\section{Conclusion}

This paper has presented the design and fabrication of a wireless, passive embedded water-content monitoring sensor for potential use in civil construction applications. This simple and cost-effective sensor was based on a single-layer spiral forming an inductive-capacitive resonant circuit due to the parasitic capacitance in the inductor. The sensor was able to detect varying amounts of water in the sample by measuring the changes in the sensor's resonant frequency. When compared to other LC sensors consisting of an inductor and capacitor or sensors based on RFIDs, the fabrication process of this sensor technology is much simpler, giving it an economical advantage. This is especially true when embedded sensors are needed to monitor large areas, thus requiring many sensors. Using three sand samples of different particle sizes and water content, the sensor showed a slower resonant frequency response at a higher water content, and vice versa. Data obtained from the tests exhibited a near linear relationship between sensor's resonant frequency and water content of the samples.

\section{References}

[1] J. S. Miller and W. Y. Bellinger, Distress Identification Manual for the Long-Term Pavement Performance Program, Publication no. FHWA-RD 03-031, FHWA, Office of Infrastructure Research and Development, McLean, Va, USA, 2003.

[2] H. Eller and A. Denoth, "A capacitive soil moisture sensor," Journal of Hydrology, vol. 185, no. 1-4, pp. 137-146, 1996.

[3] E. Grinzato, V. Vavilov, and T. Kauppinen, "Quantitative infrared thermography in buildings," Energy and Buildings, vol. 29, no. 1, pp. 1-9, 1998. 
[4] J. V. Stafford, "Remote, non-contact and in-situ measurement of soil moisture content: a review," Journal of Agricultural Engineering Research, vol. 41, no. 3, pp. 151-172, 1988.

[5] S. Nakayama, "Development of a microwave moisture sensor for aggregates," Measurement Science and Technology, vol. 6, no. 4, pp. 429-431, 1995.

[6] K. G. Ong and C. A. Grimes, "A carbon nanotube-based sensor for $\mathrm{CO}_{2}$ monitoring," Sensors, vol. 1, no. 6, pp. 193-205, 2001.

[7] K. G. Ong, J. S. Bitler, C. A. Grimes, L. G. Puckett, and L. G. Bachas, "Remote query resonant-circuit sensors for monitoring of bacteria growth: application to food quality control," Sensors, vol. 2, no. 6, pp. 219-232, 2002.

[8] K. G. Ong, J. Wang, R. S. Singh, L. G. Bachas, and C. A. Grimes, "Monitoring of bacteria growth using a wireless, remote query resonant-circuit sensor: application to environmental sensing," Biosensors and Bioelectronics, vol. 16, no. 4-5, pp. 305-312, 2001.

[9] K. G. Ong, L. G. Puckett, B. V. Sharma, M. Loiselle, C. A. Grimes, and L. G. Bachas, "Wireless, passive, resonant-circuit sensors for monitoring food quality," in Chemical and Biological Early Warning Monitoring for Water, Food, and Ground, vol. 4575 of Proceedings of SPIE, pp. 150-159, 2002.

[10] K. G. Ong, C. A. Grimes, C. L. Robbins, and R. S. Singh, "Design and application of a wireless, passive, resonant-circuit environmental monitoring sensor," Sensors and Actuators A, vol. 93, no. 1, pp. 33-43, 2001.

[11] K. G. Ong and C. A. Grimes, "A resonant printed-circuit sensor for remote query monitoring of environmental parameters," Smart Materials and Structures, vol. 9, no. 4, pp. 421-428, 2000.

[12] A. Tetelin, V. Pouget, J.-L. Lachaud, and C. Pellet, "Dynamic behavior of a chemical sensor for humidity level measurement in human breath," IEEE Transactions on Instrumentation and Measurement, vol. 53, no. 4, pp. 1262-1267, 2004.

[13] J. Laconte, V. Wilmart, D. Flandre, and J.-P. Raskin, "Highsensitivity capacitive humidity sensor using 3-layer patterned polyimide sensing film," in Proceedings of the 2nd IEEE International Conference on Sensors, vol. 1, pp. 372-377, October 2003.

[14] H. Shimizu, H. Matsumoto, M. Asakura, and K. Watanabe, "A digital hygrometer," IEEE Transactions on Instrumentation and Measurement, vol. 37, no. 2, pp. 300-304, 1988.

[15] D. Watters, P. Jayaweera, A. Bahr et al., "Wireless sensors for chloride-level monitoring in concrete bridge decks," in Structural Materials Technology V: An NDT Conference, pp. 315$320,2002$.

[16] G. P. Hammersley and M. J. Dill, "The long-term monitoring of civil engineering and building structures-developments in techniques for monitoring corrosion in reinforced and posttensioned concrete," Journal of Systems and Control Engineering, vol. 212, no. 3, pp. 175-188, 1998.

[17] M. Sun, Z. Li, Q. Liu, Z. Tang, and D. Shen, "Study on thermal self-diagnostic and self-adaptive smart concrete structures," Cement and Concrete Research, vol. 30, no. 8, pp. 1251-1253, 2000.

[18] R. Jedermann, C. Behrens, D. Westphal, and W. Lang, "Applying autonomous sensor systems in logistics: combining sensor networks, RFIDs and software agents," Sensors and Actuators A, vol. 132, no. 1, pp. 370-375, 2006.

[19] N. Wang, N. Zhang, and M. Wang, "Wireless sensors in agriculture and food industry-recent development and future perspective," Computers and Electronics in Agriculture, vol. 50, no. 1, pp. 1-14, 2006.
[20] J. Ong, Z. You, J. Mills-Beale, E. L. Tan, B. D. Pereles, and K. G. Ong, "A wireless, passive embedded sensor for real-time monitoring of water content in civil engineering materials," IEEE Sensors Journal, vol. 8, pp. 2053-2058, 2008.

[21] C. A. Balanis, Advanced Engineering Electromagnetics, John Wiley \& Sons, New York, NY, USA, 1989.

[22] A. S. Mujumar, Handbook of Industrial Drying, CRC Press, New York, NY, USA, 3rd edition, 1987. 

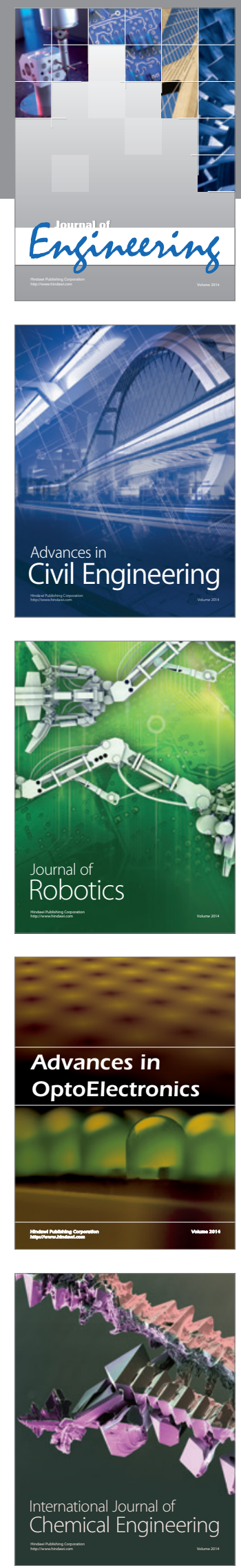

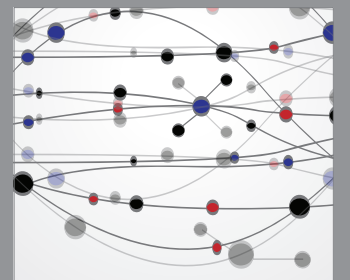

The Scientific World Journal
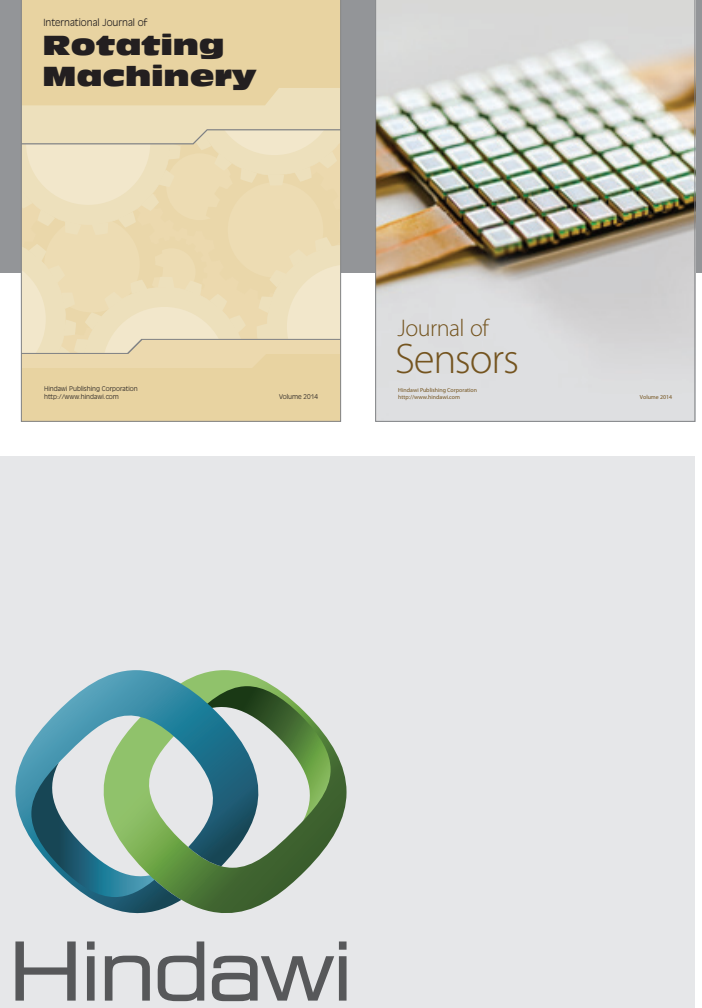

Submit your manuscripts at http://www.hindawi.com
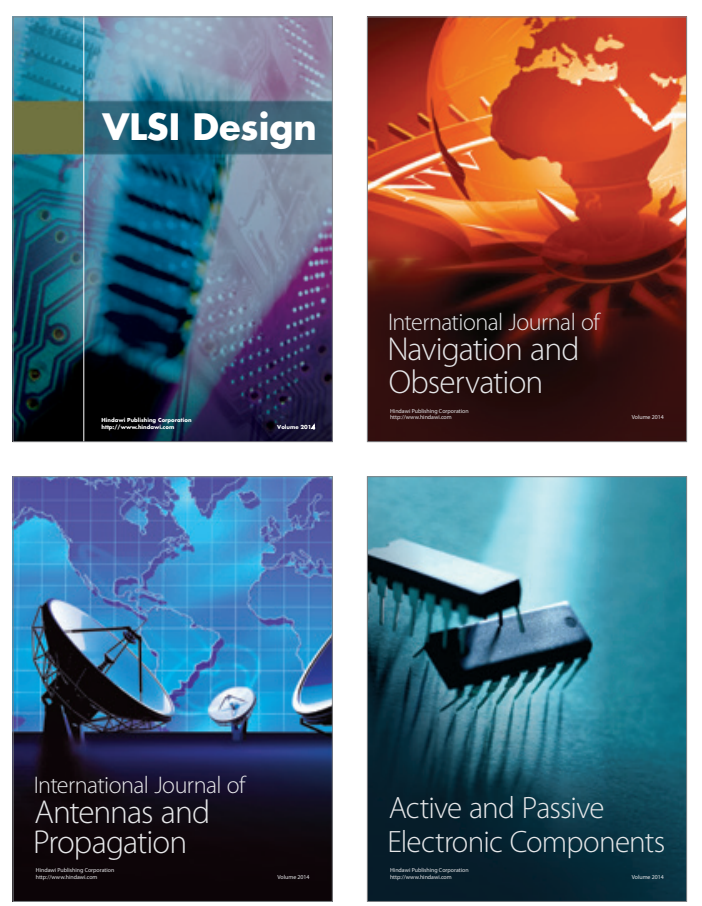
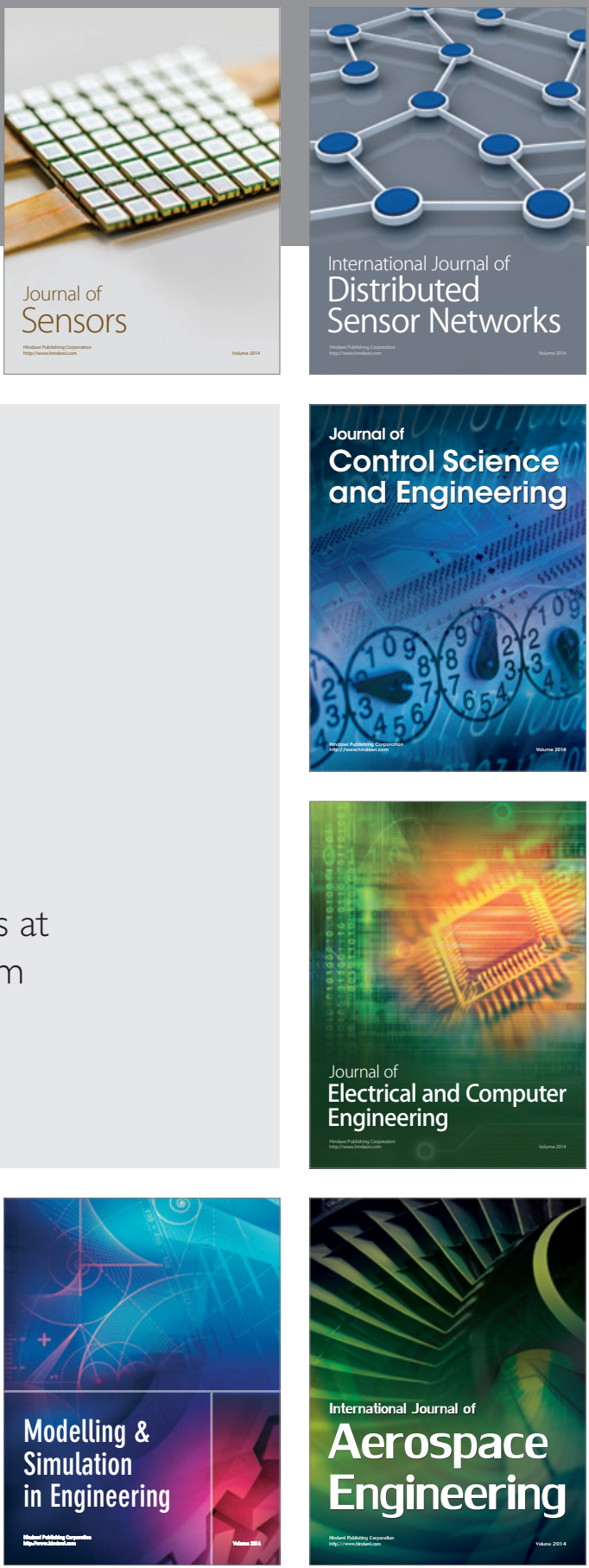

Journal of

Control Science

and Engineering
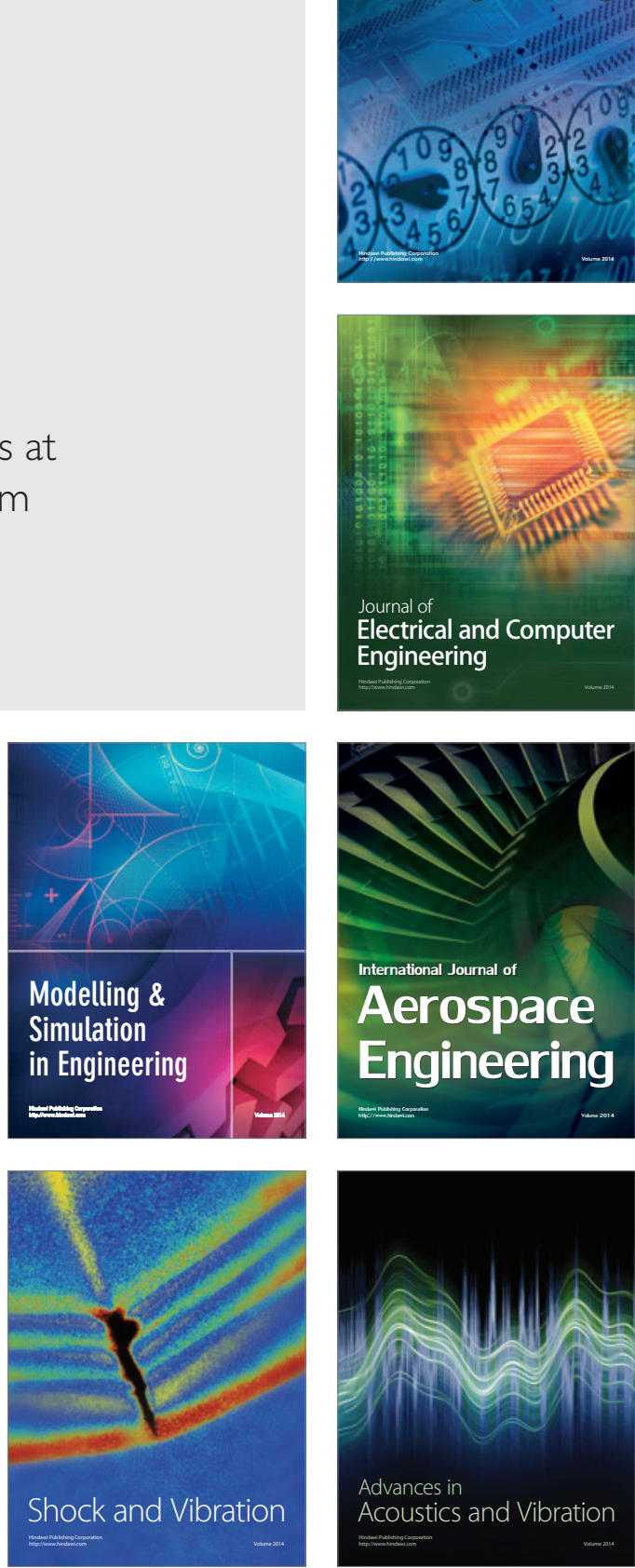